Review

\title{
Review of a Light NMSSM Pseudoscalar Higgs-State Production at the LHC
}

\author{
Mosleh M. Almarashi (D)
}

check for updates

Citation: Almarashi, M.M. Review of a Light NMSSM Pseudoscalar Higgs-State Production at the LHC. Universe 2021, 7, 392. https:// doi.org/10.3390/universe7110392

Academic Editor: Luca Fiorini

Received: 27 September 2021

Accepted: 13 October 2021

Published: 20 October 2021

Publisher's Note: MDPI stays neutral with regard to jurisdictional claims in published maps and institutional affiliations.

Copyright: ( 2021 by the author. Licensee MDPI, Basel, Switzerland. This article is an open access article distributed under the terms and conditions of the Creative Commons Attribution (CC BY) license (https://creativecommons.org/licenses/by/4.0/).
Department of Physics, Faculty of Science, Taibah University, P.O. Box 30002, Madinah 42353, Saudi Arabia; mmarashi@taibahu.edu.sa

\begin{abstract}
In this paper, we briefly review the LHC discovery potential of a light pseudoscalar Higgs boson of the NMSSM, $a_{1}$, produced in the gluon fusion $g g \rightarrow a_{1}$, bottom-quark fusion $b \bar{b} \rightarrow a_{1}$ and bottom-gluon fusion $b g \rightarrow b a_{1}$. We also review the LHC discovery potential of the next-to-lightest $\mathrm{CP}$-even Higgs boson $h_{2}$ being the non-SM-like Higgs, decaying either into two light CP-odd Higgs bosons $a_{1}$ or into a light $a_{1}$ and the $Z$ gauge boson through the gluon fusion $g g \rightarrow h_{2}$ in the $4 \tau$ final state. We find that the light $a_{1}$ can be detected at the LHC in a variety of production processes including the gluon fusion, bottom-quark fusion and bottom-gluon fusion. The latter two processes require high luminosity of the LHC and large values of $\tan \beta$. We also find that the LHC has the potential to discover the non-SM-like Higgs state, $h_{2}$, decaying into a pair of light CP-odd Higgses $a_{1}$ 's, allowing the distinguishing of the NMSSM Higgs sector from the MSSM one as such a light $a_{1}$, is impossible in the latter scenario.
\end{abstract}

Keywords: CP-odd Higgs boson; NMSSM; supersymmetric models

\section{Introduction}

The observation of the neutral Higgs boson with a mass around $125 \mathrm{GeV}$ by the ATLAS and CMS collaborations at CERN [1-4] in 2012 strongly supports the idea of supersymmetric models. In its minimal realization, the so-called Minimal Supersymmetric Standard Model (MSSM) [5,6], the SM Higgs sector is extended by introducing two Higgs doublets instead of the one in the SM. The two Higgs doublets of the MSSM give rise to five physical Higgs states: two CP-even states, $h$ and $H\left(m_{h}<m_{H}\right)$, one CP-odd state, $A$, and a pair of charged states $H^{ \pm}$instead of the only one state in the SM. However, the MSSM suffers from two critical phenomenological flaws. The first one is the $\mu$-problem [7] and the second one is a Higgs boson with a mass around $125 \mathrm{GeV}$ in the context of the MSSM requires a substantial degree of fine-tuning [8-12].

The simplest realization of supersymmetry beyond the MSSM that can solve the $\mu$-problem is the Next-to-Minimal Supersymmetric Standard Model (NMSSM) $[13,14]$. Furthermore, this model can easily accommodate the $125 \mathrm{GeV}$ SM-like Higgs mass without much fine-tuning (see for instance Refs. [15-29]). In the NMSSM one Higgs singlet superfield is added to the usual Higgs doublet ones of the MSSM. The presence of this singlet superfield leads to two additional neutral Higgs mass eigenstates and one additional neutralino mass state in the NMSSM compared to the MSSM.

The interesting phenomenological aspects of the NMSSM in comparison to the MSSM is that a light pseudoscalar Higgs boson $a_{1}$ is possible at the NMSSM. The discovery of such a light Higgs state with a mass less than the $Z$ boson mass at the LHC or other colliders would then unmistakably indicate the existence of a non-minimal SUSY Higgs sector. Such a light Higgs state can be produced at the LHC and other facilities either indirectly via decays of heavier Higgs states or directly via the gluon fusion, bottom-gluon fusion and in association with bottom quarks [30-58]. On the other hand, the experimental searches for the $a_{1}$ signal has been explored by many collaborations. No $a_{1}$ signal has been observed so far, and the results have been used to set some limits on its mass [59-68]. 
The cross sections of Higgs boson production in association with bottom-quark pair at the LHC can be calculated using two schemes, called four-flavor scheme and five-flavor scheme. In the former scheme where the bottom quarks only appear in the final states, they are not treated as partons in the protons but as massive particles. In the latter scheme where the bottom quarks are found in both initial and final states, they are treated as massless particles. Consequently, the bottom quarks are treated as partons in the protons on the same footing as other light quarks: up, down and strange, see e.g., Refs. [69,70].

The Yukawa coupling of the Higgs state to bottom-quark at the LHC can be measured by studying the interaction of bottom quarks with the Higgs field. This interaction can be probed either through the Higgs decay into bottom-antibottom quark pair or through the Higgs production in bottom-antibottom fusion. The former one is overwhelmed by very large backgrounds even though the decay of a light Higgs state to bottom quarks is dominant. Therefore, it would be helpful to study the production of the Higgs boson through bottom-quark fusion to measure the bottom-quark Yukawa coupling at the LHC. This production channel has been studied in detail in the literature [71-84].

In this paper, we will give a short review of some of the production processes that could potentially lead to the discovery of a light pseudoscalar Higgs state of the NMSSM at the LHC. We notice that such a light state can be produced at the LHC in a variety of production mechanisms, including the gluon fusion $g g \rightarrow a_{1}$, bottom-quark fusion $b \bar{b} \rightarrow a_{1}$ and bottom-gluon fusion $b g \rightarrow b a_{1}$. We also show that the process $g g \rightarrow h_{2} \rightarrow a_{1} a_{1}$ is an alternative option to produce the light pseudoscalar Higgs boson, in which case our random scan reveals that the non-SM-like Higgs boson $h_{2}$ mainly decays into a pair of $a_{1}$ 's with a branching ratio $\gtrsim 0.5$ as long as it is kinematically allowed. The content is based on our previous work [55-58]. The layout of the remainder of this article is as follows. In Section 2 we give a short review of the NMSSM, focusing on some important properties of the light pseudoscalar Higgs state $a_{1}$ inside the model and on the computation of its mass at tree-level. In Sections 3-5, we present the production rates of the light $a_{1}$ at the LHC for various production processes: the gluon fusion $g g \rightarrow a_{1}$, bottom-gluon fusion $b g \rightarrow b a_{1}$, and bottom-quark fusion $b \bar{b} \rightarrow a_{1}$, respectively. The LHC discovery potential of the light $a_{1}$ through the decays of the non-SM-like Higgs state $h_{2}$ is discussed in Section 6. Finally, Section 7 contains the conclusions.

\section{Light Pseudoscalar Higgs State $a_{1}$ in the NMSSM}

In its simplest form, the scale invariant superpotential of the NMSSM in terms of the usual two MSSM-type Higgs doublets superfields $\hat{H}_{u}$ and $\hat{H}_{d}$ together with the extra singlet superfield $\hat{S}$ takes the form

$$
W_{N M S S M}=\text { MSSM Yukawa terms }+\lambda \hat{S} \hat{H}_{u} \hat{H}_{d}+\frac{1}{3} \kappa \hat{S}^{3},
$$

where both $\lambda$ and $\kappa$ are Yukawa coupling parameters. The term $\lambda \hat{S} \hat{H}_{u} \hat{H}_{d}$ is introduced in the superpotential to solve the $\mu$-problem of the MSSM superpotential, which is the major motivation for the NMSSM. Expanding the singlet field around its vacuum expectation value $(\mathrm{VEV})\langle S\rangle=\frac{1}{\sqrt{2}} v_{S}$ dynamically generates an 'effective' $\mu$-parameter $\mu_{\text {eff }}=\lambda\langle S\rangle$ of the order of the scale of electroweak symmetry breaking. The last term of Equation (1) is added to break the Peccei-Quinn symmetry $[85,86]$. The soft breaking terms containing only the Higgs fields read

$$
V_{\mathrm{NMSSM}}=m_{H_{u}}^{2}\left|H_{u}\right|^{2}+m_{H_{d}}^{2}\left|H_{d}\right|^{2}+m_{S}^{2}|S|^{2}+\left(\lambda A_{\lambda} S H_{u} H_{d}+\frac{1}{3} \kappa A_{\kappa} S^{3}+\text { h.c. }\right),
$$

where $A_{\lambda}$ and $A_{\mathcal{K}}$ are the trilinear soft SUSY breaking parameters of the order of SUSY breaking scale $m_{\text {SUSY }}$.

Following electroweak symmetry breaking (EWSB), the physical spectrum contains seven states. In the CP conserving case (as assumed here) the NMSSM contains three 
CP-even states $h_{1,2,3}\left(m_{h_{1}}<m_{h_{2}}<m_{h_{3}}\right)$, two CP-odd states $a_{1,2}\left(m_{a_{1}}<m_{a_{2}}\right)$ and a pair of charged Higgs states $h^{ \pm}$. Due to introducing the Higgs singlet superfield, the NMSSM Higgs sector at the tree-level is described by six parameters: $\kappa, A_{\kappa}, \lambda, A_{\lambda}, \mu_{\text {eff }}$ and $\tan \beta=\frac{v_{u}}{v_{d}}$ (where $v_{u}$ and $v_{d}$ are the vacuum expectation values of the two Higgs doublets).

After the Higgs fields develop their VEVs, $\left\langle H_{u}\right\rangle=\frac{1}{\sqrt{2}} v_{u},\left\langle H_{d}\right\rangle=\frac{1}{\sqrt{2}} v_{d}$ and $\langle S\rangle=$ $\frac{1}{\sqrt{2}} v_{s}$, and eliminating the massless degree of freedom, the potential has terms for the non-zero mass modes for the scalar Higgs fields $S_{i}(i=1,2,3)$, pseudoscalar Higgs fields $P_{i}(i=1,2)$ and charged Higgs fields $h^{ \pm}$as follows

$$
V_{\text {mass }}=\frac{1}{2}\left(\begin{array}{lll}
S_{1} & S_{2} & S_{3}
\end{array}\right) \mathcal{M}_{S}\left(\begin{array}{l}
S_{1} \\
S_{2} \\
S_{3}
\end{array}\right)+\frac{1}{2}\left(\begin{array}{ll}
P_{1} & P_{2}
\end{array}\right) \mathcal{M}_{P}\left(\begin{array}{c}
P_{1} \\
P_{2}
\end{array}\right)+m_{h^{ \pm}}^{2} h^{+} h^{-} .
$$

The elements of the mass matrix for the pseudoscalar Higgs states at tree-level are [87]

$$
\begin{gathered}
\mathcal{M}_{P 11}=m_{A}^{2}, \\
\mathcal{M}_{P 12}=\frac{1}{2}\left(m_{A}^{2} \sin 2 \beta-6 \frac{\kappa \mu_{\mathrm{eff}}^{2}}{\lambda}\right) \frac{\lambda v}{\sqrt{2} \mu_{\mathrm{eff}}}, \\
\mathcal{M}_{P 22}=\frac{1}{8}\left(m_{A}^{2} \sin 2 \beta+6 \frac{\kappa \mu_{\mathrm{eff}}^{2}}{\lambda}\right) \frac{\lambda^{2} v^{2}}{\mu_{\mathrm{eff}}^{2}} \sin 2 \beta-3 \frac{\kappa \mu_{\mathrm{eff}} A_{\kappa}}{\lambda},
\end{gathered}
$$

where $m_{A}^{2}=\sqrt{2} \frac{\mu_{\text {eff }}}{\sin 2 \beta}\left(A_{\lambda}+\frac{\kappa \mu_{\text {eff }}}{\lambda}\right)$ and $v^{2}=v_{u}^{2}+v_{d}^{2}$. To a good approximation, the tree-level mass of the lightest pseudoscalar Higgs boson is given by

$$
m_{a_{1}}^{2}=\frac{9 A_{\lambda} \mu_{\mathrm{eff}}}{2 \sin 2 \beta} \cos ^{2} \theta_{A}-3 \frac{\kappa A_{\kappa} \mu_{\mathrm{eff}}}{\lambda} \sin ^{2} \theta_{A} .
$$

It is clear from the above equation that all the six tree-level parameters of the Higgs sector jointly affects $m_{a_{1}}$. In practice, one must include the various radiative corrections to the Higgs mass matrices, which affect Higgs observables [88-100].

The light pseudoscalar state $a_{1}$ is in fact a composition of both the CP-odd doublet scalar in the MSSM sector, $a_{\mathrm{MSSM}}$, and the new CP-odd singlet scalar of the NMSSM, $a_{\mathrm{S}}$. This can be written as [101]:

$$
a_{1}=a_{\mathrm{MSSM}} \cos \theta_{A}+a_{\mathrm{S}} \sin \theta_{A}
$$

where $\cos \theta_{A}$ and $\sin \theta_{A}$ are the mixing angles. The $a_{1}$ becomes highly singlet-like at small values of $\cos \theta_{A}$ as in the case of the approximate R-symmetry, in which $A_{\lambda} \rightarrow 0$ and $A_{\kappa} \rightarrow 0$ or in the case of the Peccei-Quinn symmetry, in which $\kappa \rightarrow 0$. Therefore, the NMSSM parameter space can naturally accommodate the light CP-odd Higgs $a_{1}$, which is of great interest. The $a_{1}$ could be very light, a few $\mathrm{GeV} \lesssim m_{a_{1}} \lesssim 300 \mathrm{GeV}$.

In our work to find the regions of the NMSSM parameter space that offer a light $a_{1}$, we used the NMSSMTools 5.5.2 package [102-105]. Please note that our aim is exploring the NMSSM parameter space that has a light $a_{1}$ so we assumed small values of $A_{\kappa}$. It is a numerical package calculating the masses, couplings, branching fractions and decay widths of all the Higgs bosons in addition to the SUSY spectrum of the NMSSM for each model point in terms of its input parameters. Details of our parameter space scan including theoretical and experimental constraints can be found in Refs. [55-58]. To study the LHC discovery potential of the $a_{1}$, the inclusive production rates were calculated by multiplying the production cross sections extracted from CalcHEP [106] by the branching ratios computed with the NMSSMTools for all surviving data points, assuming the center of mass energy $\sqrt{s}=14 \mathrm{TeV}$. 


\section{Light Pseudoscalar Higgs Boson Production in Gluon-Gluon Fusion at the LHC}

The light NMSSM pseudoscalar Higgs boson could be produced at the LHC via gluon fusion $g g \rightarrow a_{1}$, which is mediated by heavy quark and squark loops. This production mechanism is dominant for Higgs bosons at hadron colliders so a lot of attention has been paid to this production channel by the theory community. To study the LHC discovery potential of the $a_{1}$, we present in Figures 1 and 2 the production cross sections for the $a_{1}$ in a variety of decay channels as functions of $m_{a_{1}}$ and of the corresponding branching ratios. As expected, the production cross sections decrease with increasing $m_{a_{1}}$ as shown in the left plots of the figures. The $a_{1}$ mass for the surviving points can reach maximum about several hundreds $\mathrm{GeV}$. The production cross sections for $a_{1}$ decaying into $t \bar{t}$ are quite small, below 1 $\mathrm{fb}$ level, see the top plots of Figure 1. Therefore, for the allowed parameter space there is no point has a cross section large enough to be detectable. As for the bottom-quark pair final states, the cross sections are quite large, reaching the nanobarn (nb) level, for $m_{a_{1}} \lesssim 100$ $\mathrm{GeV}$, see the middle plots of Figure 1. It is clear that this decay channel is dominant in large region of the parameter space as shown in the right middle plot of the figure. This region corresponds to the mass range $m_{a_{1}} \lesssim 140 \mathrm{GeV}$ where the branching ratio is close to $90 \%$ for most points when the decay channel is kinematically allowed. It is also noticeable from the bottom plots of the figure that the production rates into $\tau^{+} \tau^{-}$are also sizable, reaching up to $7 \times 10^{5} \mathrm{fb}$ for very light $a_{1}$ with masses $\lesssim 10 \mathrm{GeV}$, and decreasing rapidly with increasing $m_{a_{1}}$ (left plot). Moreover, it is clear that the BR $\left(a_{1} \rightarrow \tau^{+} \tau^{-}\right)$reaches about $10 \%$ for most allowed points in the parameter space that has $m_{a_{1}} \gtrsim 10 \mathrm{GeV}$. However, there is some points with very light $a_{1}, m_{a_{1}} \lesssim 10 \mathrm{GeV}$, yielding large $\mathrm{BR}\left(a_{1} \rightarrow \tau^{+} \tau^{-}\right) \gtrsim 90 \%$, in which case the $a_{1}$ decay into $b \bar{b}$ is kinematically not allowed. The mass region with $m_{a_{1}} \lesssim 10 \mathrm{GeV}$ is highly constrained, see, e.g., Ref. [107]. These points are the ones lying in the right of the bottom-right plot of Figure 1. In short, the production rates in some regions of the NMSSM parameter space are large enough to help extracting $a_{1}$ signals in the $\tau^{+} \tau^{-}$and $b \bar{b}$ final states if the backgrounds are successfully reduced to manageable levels. However, the discovery of $a \rightarrow t \bar{t}$ is impossible because the production rates are quite small and due to the complicated final state.

In Figure 2, we show the total production cross sections for the $g g \rightarrow a_{1} \rightarrow \gamma \gamma$ and $g g \rightarrow a_{1} \rightarrow Z \gamma$ channels as functions of $m_{a_{1}}$ and of the corresponding branching ratios $\operatorname{BR}\left(a_{1} \rightarrow \gamma \gamma\right)$ and $\operatorname{BR}\left(a_{1} \rightarrow Z \gamma\right)$. One notices in the figure that the production rates reach up to about several hundreds fb for $\gamma \gamma$ and several tens fb for $Z \gamma$ final states. Such high rates correspond to $m_{a_{1}} \lesssim 200 \mathrm{GeV}$. This is interesting as such signal events could be detectable particularly at very large luminosities of the LHC. Interestingly, one also notices in Figure 3 that the $\operatorname{BR}\left(a_{1} \rightarrow \gamma \gamma\right)$ and $\operatorname{BR}\left(a_{1} \rightarrow Z \gamma\right)$ can reach unity in certain regions of the NMSSM parameter space. The reason for such dominant decay channels is that the $a_{1}$ is a singlet-like with suppressed couplings to fermions at the tree level but the $a_{1}$ can couple to $\gamma \gamma$ and $Z_{\gamma}$ at one loop with charginos in the loop. In this case, the mixing angle $\cos \theta_{A}$ gets close to 0 , see Equation (8). Unfortunately, one cannot take advantage of this phenomenon because even if the $a_{1}$ couplings to $\gamma \gamma$ and $Z_{\gamma}$ are enhanced but its couplings to gluons in the production channel are suppressed so the production cross sections are quite small when the $\operatorname{BR}\left(a_{1} \rightarrow \gamma \gamma\right)$ and the $\operatorname{BR}\left(a_{1} \rightarrow Z \gamma\right) \sim 100 \%$, see the right plots of Figure 2. The enhancements of the partial widths of $a_{1} \rightarrow \gamma \gamma$ and $a_{1} \rightarrow Z \gamma$ are characteristic features of the NMSSM in comparison to the MSSM. 

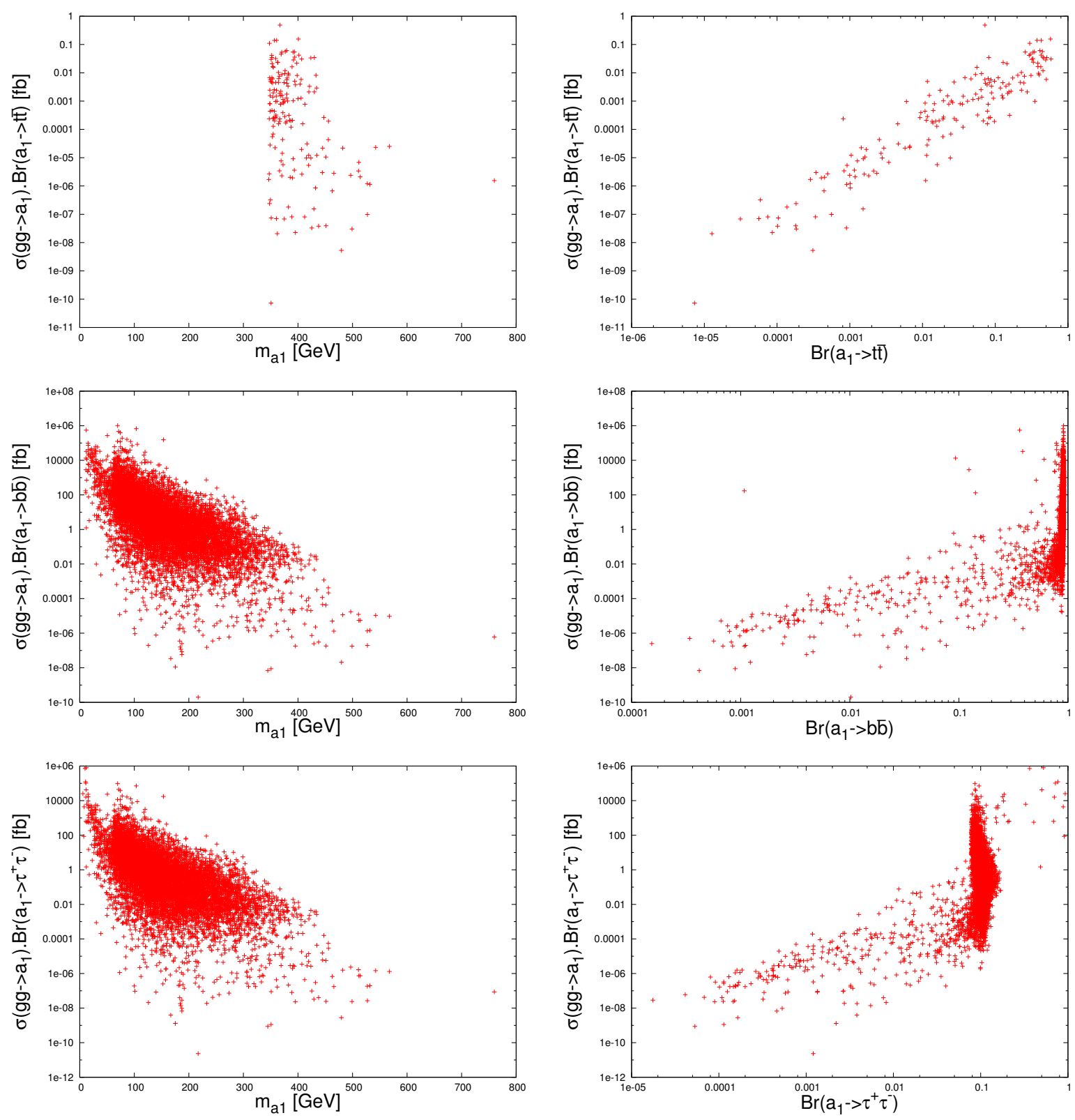

Figure 1. The production rates $\sigma\left(g g \rightarrow a_{1}\right) \operatorname{Br}\left(a_{1} \rightarrow t \bar{t}\right)($ top $) \sigma\left(g g \rightarrow a_{1}\right) \operatorname{BR}\left(a_{1} \rightarrow b \bar{b}\right)\left(\right.$ middle) and $\sigma\left(g g \rightarrow a_{1}\right) \operatorname{BR}\left(a_{1} \rightarrow \tau^{+} \tau^{-}\right)$ (bottom) as functions of $m_{a_{1}}$ (left) and of the corresponding branching ratios (right) [55]. 

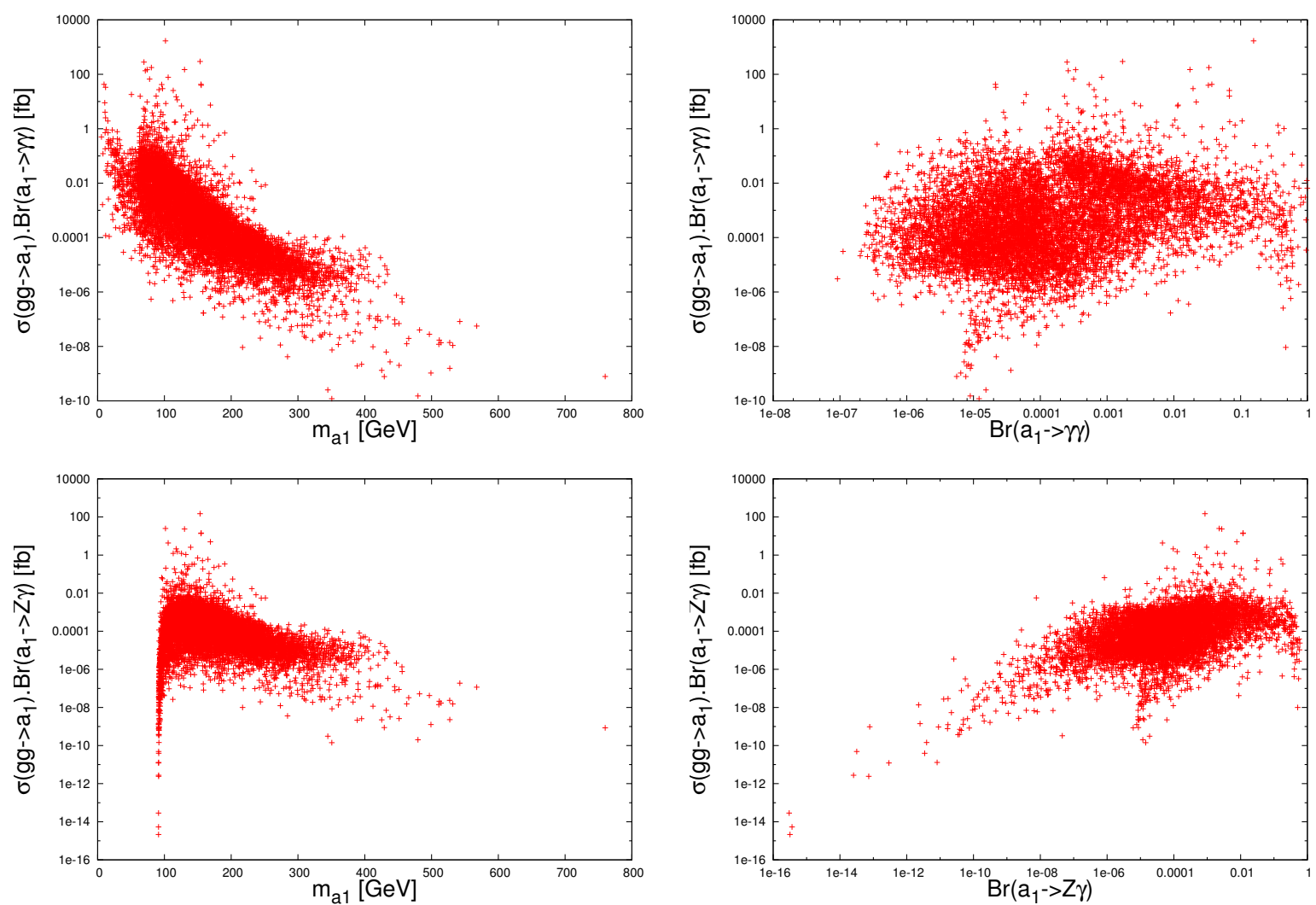

Figure 2. The production rates $\sigma\left(g g \rightarrow a_{1}\right) \operatorname{BR}\left(a_{1} \rightarrow \gamma \gamma\right)$ (top) and $\sigma\left(g g \rightarrow a_{1}\right) \operatorname{BR}\left(a_{1} \rightarrow Z \gamma\right)$ (bottom) as functions of $m_{a_{1}}($ left $)$ and of the corresponding branching ratios (right) [55].
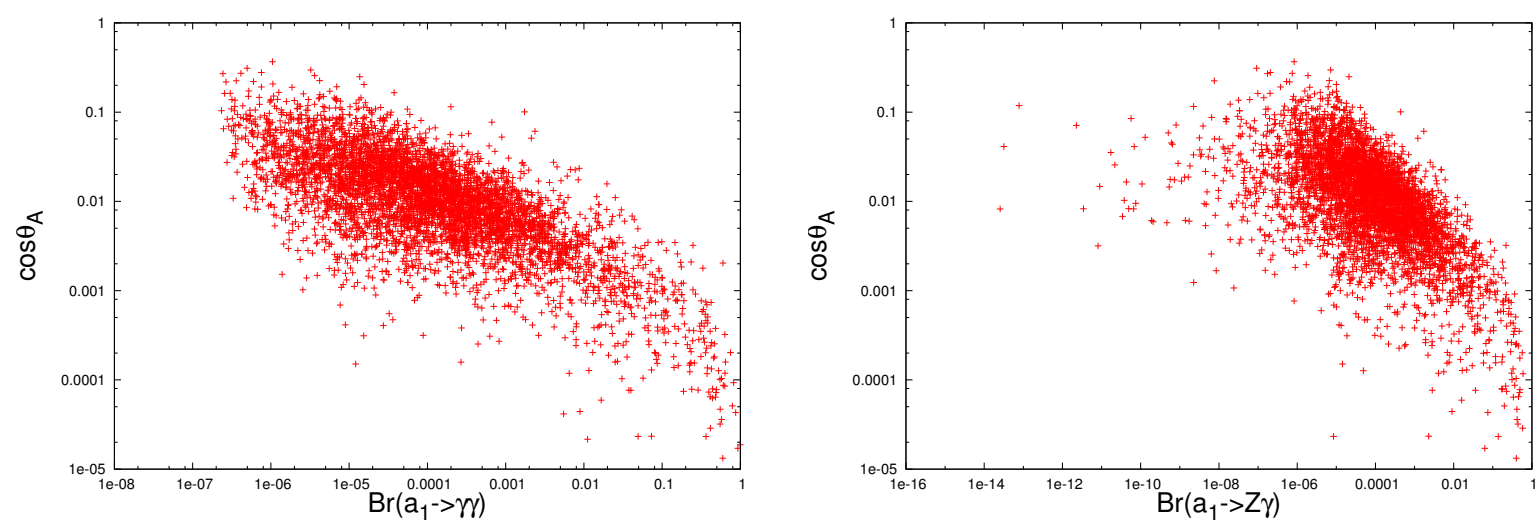

Figure 3. The correlations between the mixing angle $\cos \theta_{A}$ and the branching ratios $\operatorname{BR}\left(a_{1} \rightarrow \gamma \gamma\right)($ left $)$ and $\operatorname{BR}\left(a_{1} \rightarrow Z \gamma\right)($ right $)[55]$.

\section{Light Pseudoscalar Higgs Boson Production with a Single Bottom-Quark at the LHC}

The $a_{1}$ production in association with a single bottom-quark at hadron colliders can occur at the tree-level through either the process $g b \rightarrow b a_{1}$ or the process $g \bar{b} \rightarrow \bar{b} a_{1}$. Since the production cross sections for the process $p p \rightarrow b a_{1}$ at the LHC and its charge conjugate $p p \rightarrow a_{1} \bar{b}$ are alike, we only consider here the production process $p p \rightarrow a_{1} b$. This process has an advantage that the existence of the bottom-quark with a high transverse momentum in the final state can be tagged, allowing the rejection of light jets. Furthermore, this process can be significantly enhanced at large values of $\tan \beta$. Since the $a_{1}$ production through the process $p p \rightarrow a_{1} b$ followed by the $a \rightarrow b \bar{b}$ decay is plagued by large backgrounds, we only discuss in this section the LHC discovery potential of a light pseudoscalar through the process $p p \rightarrow b a_{1}$ followed by the $a_{1} \rightarrow \tau^{+} \tau^{-}$decay. 
Figure 4 shows the distribution of the production rates $\sigma\left(g b \rightarrow a_{1}\right) \operatorname{BR}\left(a_{1} \rightarrow \tau^{+} \tau^{-}\right)$as functions of $m_{a_{1}}$ and of $\operatorname{BR}\left(a_{1} \rightarrow \tau^{+} \tau^{-}\right)$. These rates decreases rapidly with increasing $m_{a_{1}}$ as shown in the left plot of the figure. The production rates can reach up to $10 \mathrm{nb}$ for $\operatorname{BR}\left(a_{1} \rightarrow \tau^{+} \tau^{-}\right) \approx 0.1$ and $25 \mathrm{nb}$ for $\operatorname{BR}\left(a_{1} \rightarrow \tau^{+} \tau^{-}\right) \approx 1$ as shown in the right plot of the figure. In short, it is remarkable to notice that these production rates are quite sizable for considerable points in the NMSSM parameter space, which can reach detectable levels.
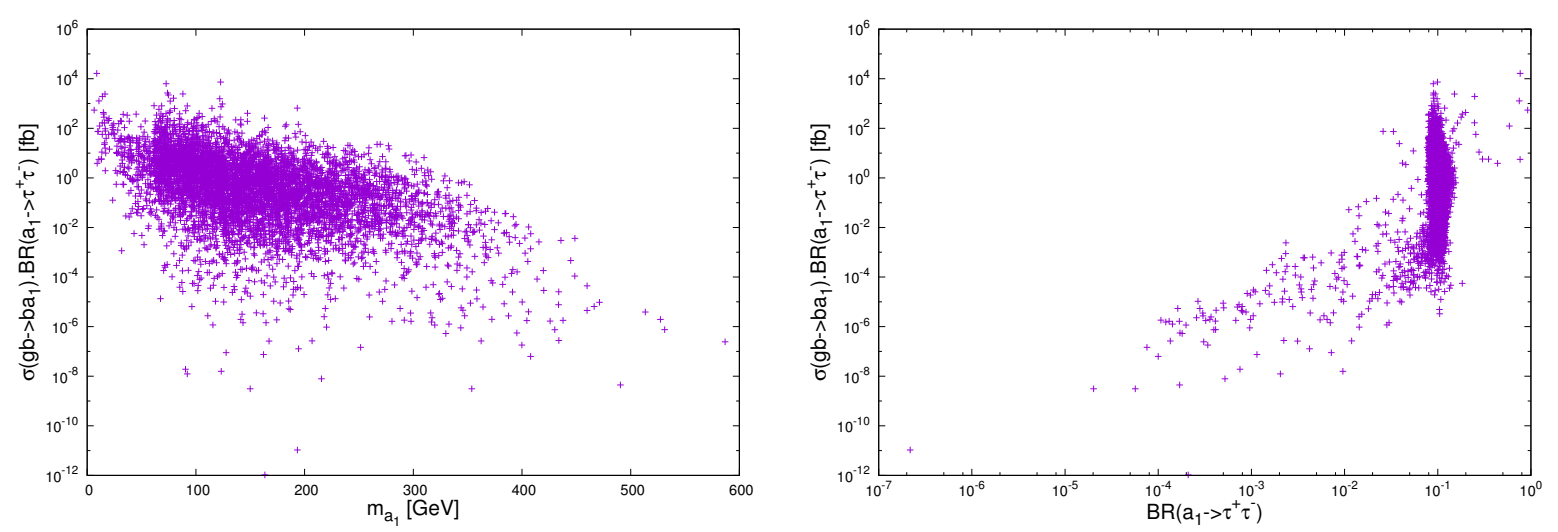

Figure 4. The production rates $\sigma\left(g g \rightarrow a_{1}\right) \operatorname{BR}\left(a_{1} \rightarrow \tau^{+} \tau^{-}\right)$as functions of both $m_{a_{1}}(\mathbf{l e f t})$ and $\operatorname{BR}\left(a_{1} \rightarrow \tau^{+} \tau^{-}\right)($right $)[56]$.

A partonic signal-to-background (S/B) analysis for the process $p p \rightarrow a_{1} b \rightarrow b \tau^{+} \tau^{-}$ was done in Ref. [56] where extraction of the $a_{1}$ signature was proven for several benchmark points given in Table 1 . Figure 5 shows the signal significance $S / \sqrt{B}$ (where $S$ and $B$ are the signal and background rates, respectively), left plot, and the corresponding signal event rate $S$, right plot, as functions of the integrated luminosity. One can see that the discovery of the light $a_{1}$ at the LHC may occur for $a_{1}$ masses between $\approx 90$ and $\approx 150 \mathrm{GeV}$ at a luminosity of $\mathcal{O}\left(300 \mathrm{fb}^{-1}\right)$ or more where the signal significances, $S / \sqrt{B}$, are good enough to discover the $a_{1}$ in this mass region.

Table 1. Four benchmark points used in the signal-to-background (S/B) analysis [56].

\begin{tabular}{cccc}
\hline Point & $\lambda$ & $\kappa$ & $\tan \beta$ \\
\hline 1 & 0.17633 & 0.248102 & 15.9481 \\
2 & 0.295035 & 0.481850 & 17.2552 \\
3 & 0.259318 & -0.548899 & 13.4015 \\
4 & 0.120601 & 0.294640 & 13.2896 \\
\hline Point & $\mu_{\mathrm{eff}}[\mathrm{GeV}]$ & $A_{\lambda}[\mathrm{GeV}]$ & $A_{\kappa}[\mathrm{GeV}]$ \\
\hline 1 & 165.357 & 34.9856 & 0.0221315 \\
2 & 169.105 & -15.7234 & -2.63603 \\
3 & 177.252 & $540.586 \mathrm{E}$ & 16.2739 \\
4 & 766.903 & -1821.2 & -8.76219 \\
\hline Point & $\mathrm{m}_{\mathrm{a}_{1}}[\mathrm{GeV}]$ & $\mathrm{BR}\left(\mathrm{a}_{1} \rightarrow \tau^{+} \tau^{-}\right)$ & $\sigma\left(\mathrm{gb} \rightarrow b a_{1}\right) . B R\left(a_{1} \rightarrow \tau^{+} \tau^{-}\right)[\mathrm{fb}]$ \\
\hline 1 & 8.80412 & 0.767892 & $16,340.7$ \\
2 & 45.0156 & 0.0808669 & 371.75 \\
3 & 92.0510 & 0.0929458 & 796.7 \\
4 & 147.483 & 0.108216 & 400.9 \\
\hline
\end{tabular}



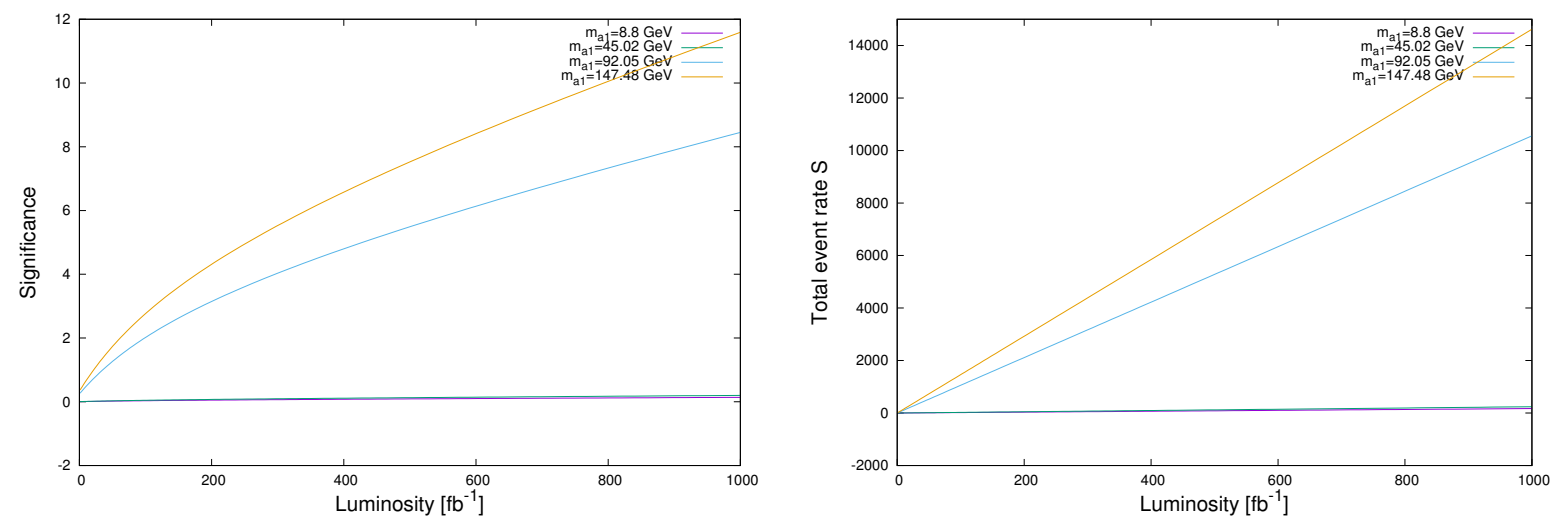

Figure 5. The signal significance $S / \sqrt{B}$ (left plot) and the signal event rate $S$ (right plot) of the process $b g \rightarrow a_{1} b \rightarrow b \tau^{+} \tau^{-}$as functions of the integrated luminosity for the four benchmark points mentioned in Table 1 . The lines for both $m_{a_{1}}=8.8 \mathrm{GeV}$ and $45.02 \mathrm{GeV}$ may be hardly visible but they were added for clarity.

\section{Light Pseudoscalar Higgs Boson Production in Bottom-Quark Annihilation at the LHC}

Higgs production in association with bottom quarks in supersymmetric theories with large $\tan \beta$ can be the dominant Higgs production mechanism in hadronic collisions as the bottom Yukawa coupling can be enhanced compared to that of the SM. This enhancement of the bottom Yukawa coupling in supersymmetric theories occurs only at large $\tan \beta$. As mentioned in the introduction, the cross sections for this process can be calculated using either the four-flavor scheme where the leading order processes is $p p \rightarrow b \bar{b} H$ or using the five-flavor scheme where the leading order one is $b \bar{b} \rightarrow H$.

In this section, we consider the direct production of a light $a_{1}$ through the process $b \bar{b} \rightarrow$ $a_{1}$ in the context of the NMSSM rather than looking for its traditional production $p p \rightarrow b \bar{b} a_{1}$, which has been studied in the literature [42-44]. Figure 6 presents the production cross sections $\sigma\left(b \bar{b} \rightarrow a_{1}\right) \operatorname{BR}\left(a_{1} \rightarrow \tau^{+} \tau^{-}\right)$as functions of $\tan \beta$ (top plot), $m_{a_{1}}$ (middle plot) and $\operatorname{BR}\left(a_{1} \rightarrow \tau^{+} \tau^{-}\right)$(bottom plot) for all the surviving points of the scan. We can see from the figure that the production rates are sizable, topping $10^{9} \mathrm{pb}$. These largest rates correspond to the region of large values of $\tan \beta$ in which the $a_{1}$ is a light, $m_{a_{1}} \lesssim 200 \mathrm{GeV}$. The bottom plot of the figure shows the dependence of the production rates on the $\operatorname{BR}\left(a_{1} \rightarrow \tau^{+} \tau^{-}\right)$. It is clear that the population density of the points corresponds to the region of $\operatorname{BR}\left(a_{1} \rightarrow \tau^{+} \tau^{-}\right)$ $\sim 10 \%$. In summary, the $a_{1}$ production in bottom-quark annihilation in the tauonic decay channel at the LHC seems to be a promising production channel for the discovery of the light $a_{1}$ in the region of large values of $\tan \beta$. Some scope could also be afforded by $\gamma \gamma$ signature in this production channel, see Ref. [108]. 

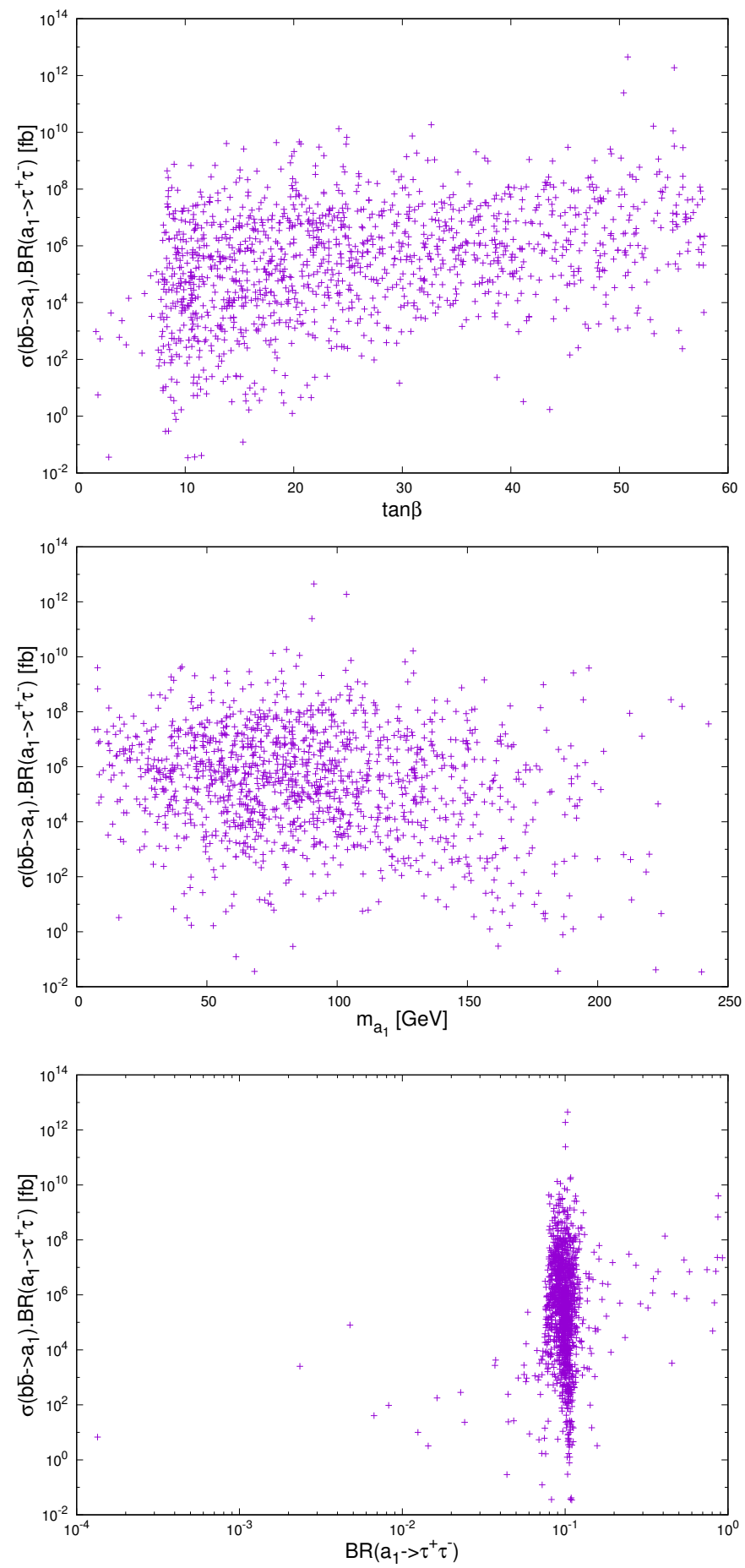

Figure 6. Production rates for the lightest pseudoscalar Higgs state, $\sigma\left(b \bar{b} \rightarrow a_{1} \rightarrow \tau^{+} \tau^{-}\right)$, as functions of $\tan \beta$ (top), $m_{a_{1}}$ (middle) and $\operatorname{BR}\left(a_{1} \rightarrow \tau^{+} \tau^{-}\right)$(bottom) [57].

Several analyses for signals and dominant backgrounds for the process $b \bar{b} \rightarrow a_{1} \rightarrow$ $\tau^{+} \tau^{-}$were done for the six benchmark points given in Table 2, see Ref. [57] for more details. Figure 7 shows the signal significance and the signal event rates for these benchmark points. As it can be seen from this figure, the LHC has the potential to observe the $a_{1}$ signal in the range $150 \lesssim m_{a_{1}} \lesssim 200 \mathrm{GeV}$ but this requires very high luminosity of $600 \mathrm{fb}^{-1}$ or more, see the left plot of the figure. Lighter or Heavier states could not be resolvable via this production channel. 
Table 2. The benchmark points used in the signal-to-background analysis for the process $b \bar{b} \rightarrow a_{1} \rightarrow \tau^{+} \tau^{-}[$[57].

\begin{tabular}{ccccccr}
\hline & P1 & P2 & P3 & P4 & P5 & P6 \\
\hline$\lambda$ & 0.0549371 & 0.248102 & 0.217092 & 0.197646 & 0.172677 & 0.249246 \\
\hline$\kappa$ & 0.496695 & 0.618832 & 0.392990 & -0.349694 & 0.481966 & 0.547502 \\
\hline $\tan \beta$ & 13.8036 & 18.8314 & 32.9745 & 53.9098 & 20.6563 & 12.2270 \\
\hline$\mu_{\mathrm{eff}}[\mathrm{GeV}]$ & 106.941 & 139.910 & 328.891 & 546.213 & 622.326 & 875.295 \\
\hline$A_{\lambda}[\mathrm{GeV}]$ & 490.984 & 826.568 & 339.250 & 1125.35 & -1434.74 & -1201.27 \\
\hline$A_{\kappa}[\mathrm{GeV}]$ & -0.0759634 & -0.310352 & -4.45516 & 4.52072 & -4.38217 & -4.45711 \\
\hline $\mathrm{m}_{\mathrm{a}_{1}}[\mathrm{GeV}]$ & 8 & 52 & 100 & 150 & 197 & 243 \\
\hline $\mathrm{BR}\left(\mathrm{a}_{1} \rightarrow \tau^{+} \tau^{-}\right)$ & 0.870856 & 0.0835167 & 0.103431 & 0.121666 & 0.116006 & 0.118482 \\
\hline$\sigma[\mathrm{fb}]$ & $4 \times 10^{9}$ & $5.1 \times 10^{8}$ & $3 \times 10^{7}$ & $4.8 \times 10^{7}$ & $3.9 \times 10^{9}$ & $3.6 \times 10^{7}$ \\
\hline
\end{tabular}
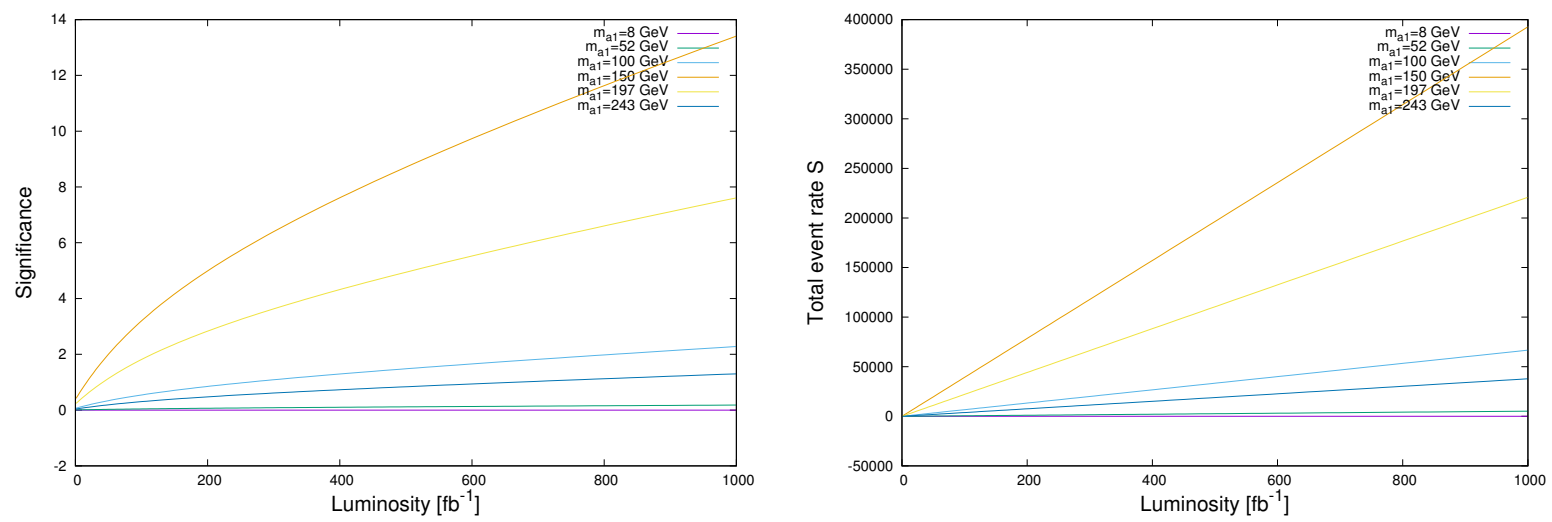

Figure 7. The signal significance $S / \sqrt{B}$ (left plot) and the total event rate $S$ (right plot) of the production process $b \bar{b} \rightarrow a_{1} \rightarrow \tau^{+} \tau^{-}$as functions of the integrated luminosity for the six benchmark points mentioned in Table 2.

\section{Light Pseudoscalar Higgs Boson Production via Decays of a Heavy Scalar Higgs Boson at the LHC}

In this section, we consider the production of the light $a_{1}$ through the non-SM Higgs boson, $h_{2}$, decaying either into two light CP-odd Higgs bosons $a_{1} a_{1}$ or into a light $a_{1}$ and the gauge boson Z. One of the interesting features of the NMSSM is the fact that Higgs-toHiggs decays can be kinematically open in large areas of the parameter space in which the existence of the light $a_{1}$ is quite natural, see e.g., Refs. [30-33]. Here, we only consider the gluon fusion $g g \rightarrow h_{2}$ followed by either $a_{1} a_{1}$ or and $Z a_{1}$ as a potential production channel to produce the light $a_{1}$.

Figure 8 shows the correlations between $m_{h_{2}}$ and the lightest CP-even and CP-odd Higgs masses $m_{h_{1}}$ and $m_{a_{1}}$ for the good points. Since the surviving points emerging from the random scan have large values of $\lambda$ and small values of both $\mu_{\text {eff }}$ and $A_{\kappa}$, only small values of $m_{h_{2}}$ and $m_{a_{1}}$ are allowed, see Ref. [58]. It is remarkable to notice that in most regions of our parameter space the $h_{1}$ is the SM-like Higgs boson of the mass around $125 \mathrm{GeV}$. However, the $h_{2}$ can also play the role of the SM-like Higgs boson but only in small regions of the parameter space in which regions the $h_{1}$ is mostly singlet-like with mass $\lesssim 120 \mathrm{GeV}$, see the left-plot of the figure, so it can escape the LEP, the Tevatron, and LHC bounds [109-115]. The right plot of the figure shows that the smaller the $m_{h_{2}}$, the smaller the $m_{a_{1}}$.

Due to the mixing between the Higgs singlet and doublets, Higgs-to-Higgs decays can be dominant over large regions of the parameter space if these decays are kinematically allowed. In Figure 9 we display the correlations between the $h_{2} \rightarrow a_{1} a_{1}$ decay rate and 
the $m_{h_{2}}$ (left) and the correlations between the $h_{2} \rightarrow Z a_{1}$ decay rate and the $m_{h_{2}}$ (right). It is clear from the left plot of the figure that the decay $h_{2} \rightarrow a_{1} a_{1}$ is dominant, reaching unity, whenever it is kinematically allowed. The dominance of this decay mode causes a suppression of the other decay channels such as $b \bar{b}$. It is also noticed that the $h_{2} \rightarrow Z a_{1}$ branching ratio is quite small, topping $0.1 \%$ as shown from the right plot of the figure.
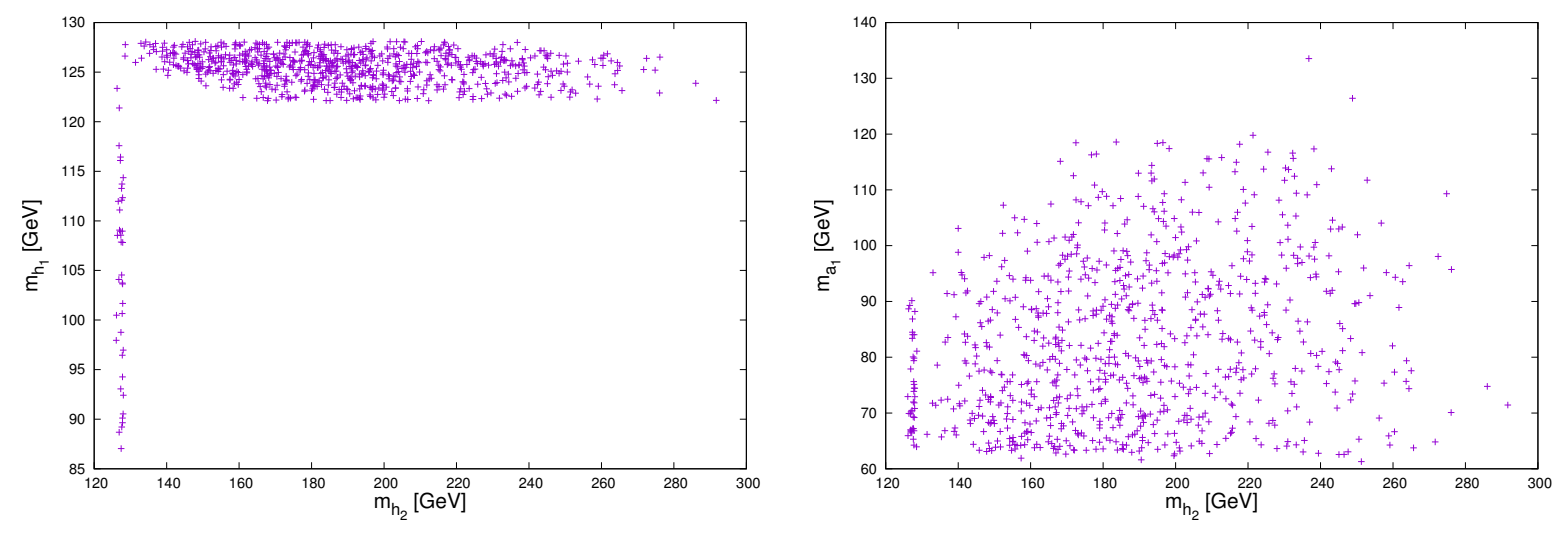

Figure 8. The mass distribution for the next-to-lightest CP-even Higgs, $m_{h_{2}}$, versus the lightest CP-even Higgs mass $m_{h_{1}}($ left plot) and versus the lightest CP-odd Higgs mass $m_{a_{1}}$ (right plot) [58].
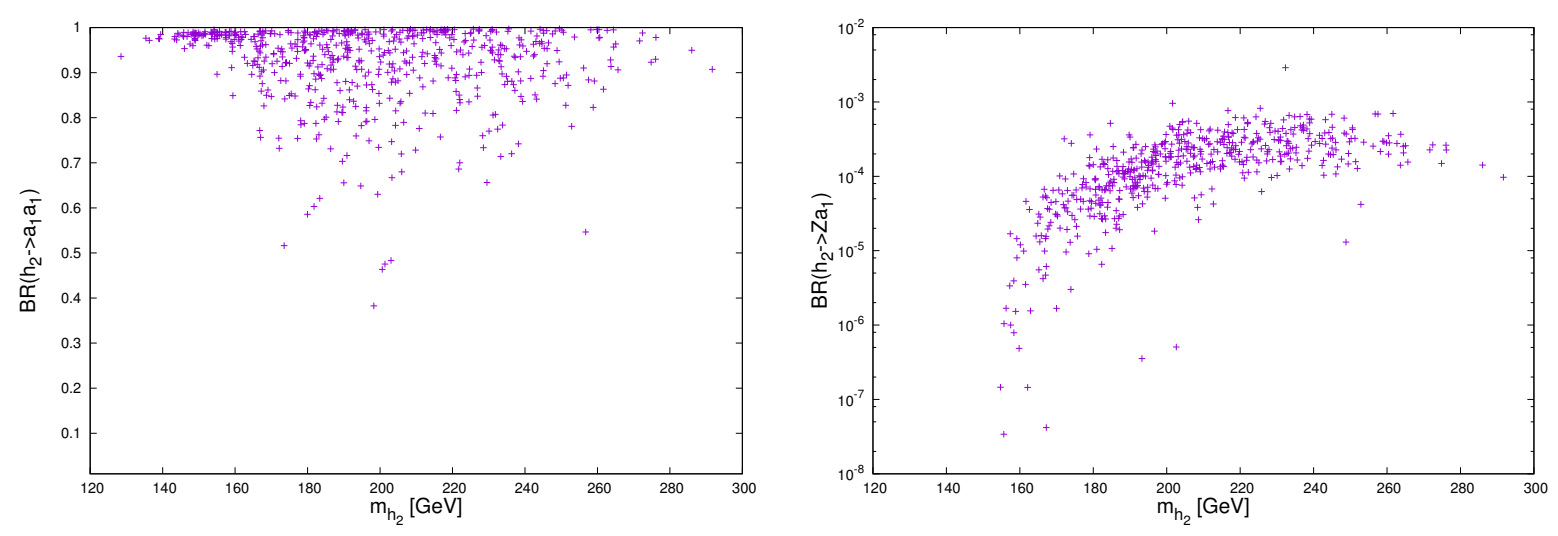

Figure 9. The next-to-lightest CP-even Higgs mass $m_{h_{2}}$ plotted against both $\mathrm{BR}\left(h_{2} \rightarrow a_{1} a_{1}\right)(\mathbf{l e f t})$ and $\mathrm{BR}\left(h_{2} \rightarrow Z a_{1}\right)($ right $)[58]$.

Furthermore, by Looking at the inclusive production rates ending up with $a_{1} a_{1} \rightarrow$ $\tau^{+} \tau^{-} \tau^{+} \tau^{-}$and $Z a_{1} \rightarrow \tau^{+} \tau^{-} \tau^{+} \tau^{-}$in Figure 10, it is clear that these rates decrease by increasing $m_{h_{2}}$, as expected. Additionally, it is clear that the $\sigma\left(g g \rightarrow h_{2} \rightarrow a_{1} a_{1} \rightarrow\right.$ $\left.\tau^{+} \tau^{-} \tau^{+} \tau^{-}\right)$are sizable, reaching a maximum level at about $100 \mathrm{fb}$ for small values of $m_{h_{2}}$ while the production rates $\sigma\left(g g \rightarrow h_{2} \rightarrow Z a_{1} \rightarrow \tau^{+} \tau^{-} \tau^{+} \tau^{-}\right)$are definitely too small, reaching its maximum of roughly $0.5 \mathrm{fb}$. The latter production rates are obviously not enough to detect the $a_{1}$ through this channel at the LHC, assuming that leptonic tau decays are around $17.5 \%$. We do not consider the $b \bar{b} b \bar{b}$ and $b \bar{b} \tau^{+} \tau^{-}$final states here because they are plagued by large backgrounds.

After performing analyses for signals and dominant standard model backgrounds in Ref. [58] for the four benchmark points shown in Table 3 for the process $g g \rightarrow h_{2} \rightarrow a_{1} a_{1} \rightarrow$ $\tau^{+} \tau^{-} \tau^{+} \tau^{-}$, we conclude that there are some regions of the NMSSM parameter space where the $h_{2}$ and $a_{1}$ could spontaneously be discovered in the mass region $140 \lesssim m_{h_{2}} \lesssim 220 \mathrm{GeV}$ and $m_{a_{1}} \lesssim 100 \mathrm{GeV}$ at the LHC of $1000 \mathrm{fb}^{-1}$ of luminosity. The existence of such a light $a_{1}$ is an exciting prospect to distinguish the NMSSM from the MSSM as such a light pseudoscalar Higgs state, about $M_{Z}$ or below in mass, is impossible in the MSSM. 

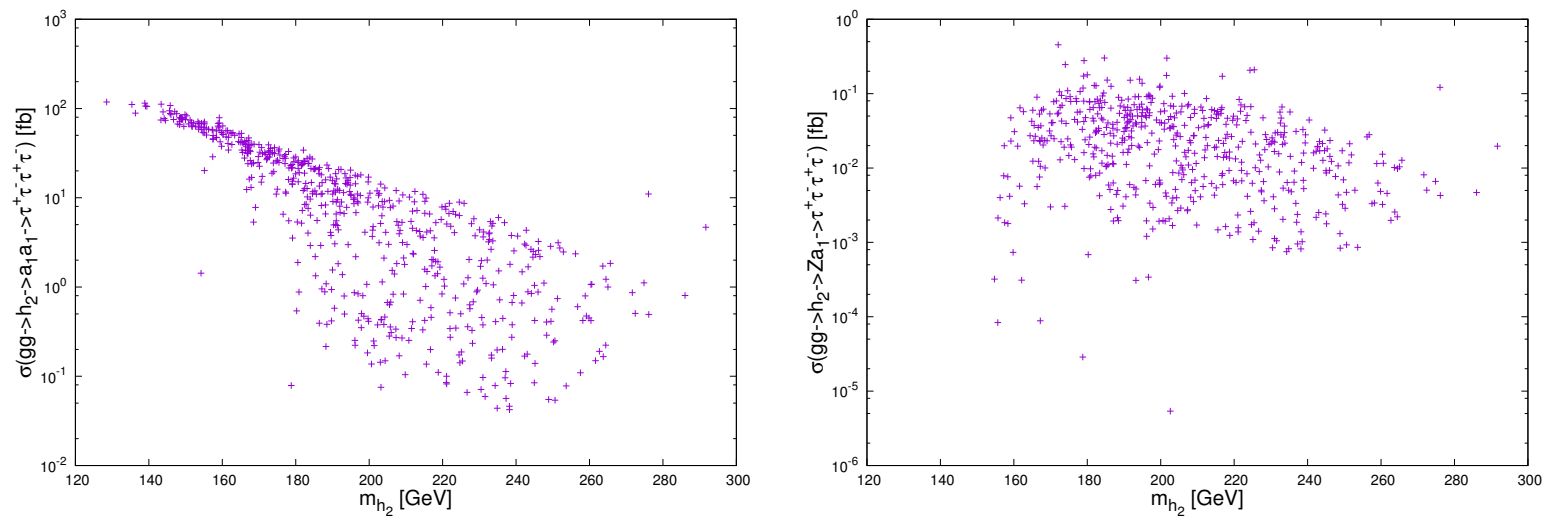

Figure 10. The production rates $\sigma\left(g g \rightarrow h_{2}\right) . \mathrm{BR}\left(h_{2} \rightarrow a_{1} a_{1} \rightarrow \tau^{+} \tau^{-} \tau^{+} \tau^{-}\right)(\mathbf{l e f t})$ and $\sigma\left(g g \rightarrow h_{2}\right) . \mathrm{BR}\left(h_{2} \rightarrow Z a_{1} \rightarrow \tau^{+} \tau^{-} \tau^{+} \tau^{-}\right)$ (right) as functions of $m_{h_{2}}$ [58].

Table 3. The four benchmark points P1, P2, P3, and P4 used in the signal-to-backgrounds analysis [58].

\begin{tabular}{ccccc}
\hline & P1 & P2 & P3 & P4 \\
\hline$\lambda$ & 0.615706 & 0.650828 & 0.637590 & 0.617789 \\
\hline$\kappa$ & 0.261287 & 0.264725 & 0.339134 & 0.387478 \\
\hline $\tan \beta$ & 5.2247 & 3.78738 & 3.82514 & 3.70979 \\
\hline$\mu_{\mathrm{eff}}[\mathrm{GeV}]$ & 153.678 & 198.766 & 198.201 & 199.224 \\
\hline$A_{\lambda}[\mathrm{GeV}]$ & 646.778 & 517.464 & 464.215 & 426.835 \\
\hline$A_{\kappa}[\mathrm{GeV}]$ & -8.00937 & 5.1126 & -9.72344 & 9.09329 \\
\hline $\mathrm{m}_{\mathrm{h}_{2}}[\mathrm{GeV}]$ & 140 & 180 & 220 & 260 \\
\hline $\mathrm{m}_{\mathrm{a}_{1}}[\mathrm{GeV}]$ & 66 & 64 & 99 & 67 \\
\hline$S[\mathrm{fb}]$ with $300 \mathrm{fb}^{-1}$ & $3.168 \times 10^{4}$ & $8.61 \times 10^{3}$ & $2.364 \times 10^{3}$ & $3.21 \times 10^{2}$ \\
\hline$B[\mathrm{fb}]$ with $300 \mathrm{fb}^{-1}$ & $3.9 \times 10^{4}$ & $3.9 \times 10^{4}$ & $3.9 \times 10^{4}$ & $3.9 \times 10^{4}$ \\
\hline$S / \sqrt{B}$ with $300 \mathrm{fb}^{-1}$ & 160.4 & 43.6 & 12 & 1.6 \\
\hline$S / \sqrt{B}$ with $1000 \mathrm{fb}^{-1}$ & 292.9 & 79.6 & 21.9 & 3 \\
\hline
\end{tabular}

\section{Conclusions}

The NMSSM can naturally accommodate the measured value of the SM-like-Higgs boson mass of the range $\sim 125 \mathrm{GeV}$ without a significant degree of fine-tuning as compared to the MSSM. In the CP conserving case, the Higgs sector of the NMSSM consists of seven physical Higgs mass states: three CP-even, two CP-odd and two charged states. Noticeably, the discovery of any of the light Higgs states along with the SM-like-Higgs state discovered in 2012 would be a clear signature of the non-minimal supersymmetric models. In this paper, in the framework of the NMSSM, we have reviewed the LHC discovery potential of a light pseudoscalar Higgs, $a_{1}$, in light of experimental and theoretical constraints implemented in the program NMSSMTools apart from the dark matter and the muon anomalous magnetic moment constraints, focusing on the following Higgs production channels: gluon fusion $g g \rightarrow a_{1}$, bottom-gluon fusion $b g \rightarrow b a_{1}$, bottom-quark fusion $b \bar{b} \rightarrow a_{1}$ in addition to the production of $a_{1}$ via decays of the non-SM-like Higgs boson: $g g \rightarrow h_{2} \rightarrow a_{1} a_{1}$ and $g g \rightarrow h_{2} \rightarrow Z a_{1}$. Under the given results, we have concluded that:

1. In the gluon fusion production channel, the overall production rates are quite sizable and could help extracting the $a_{1}$ signal over some parts of the parameter space. We believe that the most promising decay channels for the $a_{1}$ discovery at the LHC are the $\tau^{+} \tau^{-}$and $\gamma \gamma$ channels. The other promising channels are the $b \bar{b}$ and $Z \gamma$ channels 
but the former one suffers from large backgrounds and one should successfully reduce them to manageable levels. As for the latter one, there is only a small but well-defined region of the NMSSM parameter space where the light $a_{1}$ could be discovered through this decay channel.

2. It has also been noted that at high luminosity of the LHC the $\tau^{+} \tau^{-}$decay channel can be exploited to discover the light $a_{1}$ produced with a single bottom-quark $b g \rightarrow b a_{1}$ in the mass region $90 \lesssim m_{a_{1}} \lesssim 150 \mathrm{GeV}$.

3. The production of the light pseudoscalar Higgs boson in the bottom-quark fusion $b b \rightarrow a_{1}$ followed by the decay channel $a_{1} \rightarrow \tau^{+} \tau^{-}$is also possible at large values of $\tan \beta$ in the mass region $150 \lesssim m_{a_{1}} \lesssim 200 \mathrm{GeV}$, provided that the luminosity of $\mathcal{O}\left(600 \mathrm{fb}^{-1}\right)$ or more.

In the above three production channels, we have found that the $\tau$-pair decay is a promising channel to discover the light $a_{1}$ at the LHC. Here, we assume that one $\tau$ decays leptonically and the other decays hadronically. This is because they combine the high efficiency of the lepton trigger and the largeness of the branching fractions of the hadronic tau decays. The case of fully hadronic decays is ignored due to the huge QCD backgrounds.

4. Furthermore, the $a_{1}$ production through the process $g g \rightarrow h_{2} \rightarrow a_{1} a_{1}$ in the $4 \tau$ final state affords the possibility to discover the light $a_{1}$ in the mass regions $140 \lesssim m_{h_{2}} \lesssim 220 \mathrm{GeV}$ and $m_{a_{1}} \lesssim 100 \mathrm{GeV}$. We have found that this production channel can give sizable production rates, which could allow one to discover simultaneously two Higgs bosons: the nonSM-like Higgs boson, $h_{2}$, and the light pseudoscalar Higgs state, $a_{1}$. However, the $a_{1}$ production of the process $g g \rightarrow h_{2} \rightarrow Z a_{1} \rightarrow 4 \tau$ is impossible due to the smallness of the production rates. Finally, we have noticed in our choice of the NMSSM parameter space that the $\operatorname{BR}\left(h_{2} \rightarrow a_{1} a_{1}\right)$ is dominant in most regions of the parameter space as long as it is kinematically allowed.

Overall, searching for a light pseudoscalar Higgs state at the LHC and other colliders is of great value as many models predict the existence of such a light state. We hope that our encouraging results motivate further and more detailed studies in the context of the NMSSM since it offers a much richer accessible phenomenology of the light pseudoscalar state that deserves to be comprehensively exploited.

Funding: This work is supported by Taibah University in Saudi Arabia.

Institutional Review Board Statement: Not applicable.

Informed Consent Statement: Not applicable.

Data Availability Statement: Not applicable.

Acknowledgments: The author acknowledges support of Taibah University.

Conflicts of Interest: The author declares no conflict of interest.

\section{References}

1. Chatrchyan, S. et al. [CMS Collaboration]. Observation of a new boson at a mass of $125 \mathrm{GeV}$ with the CMS experiment at the LHC. Phys. Lett. B 2012, 716, 30.

2. Aad, G. et al. [ATLAS Collaboration]. Observation of a new particle in the search for the Standard Model Higgs boson with the ATLAS detector at the LHC. Phys. Lett. B 2012, 716, 1. [CrossRef]

3. Chatrchyan, S. et al. [CMS Collaboration]. Observation of a new boson with mass near $125 \mathrm{GeV}$ in pp collisions at $\sqrt{s}=7$ and 8 TeV. J. High Energy Phys. 2013, 1306, 081.

4. Aad, G. et al. [ATLAS Collaboration]. Measurements of Higgs boson production and couplings in diboson final states with the ATLAS detector at the LHC. Phys. Lett. B 2013, 726, 88.

5. Martin, S.P. A Supersymmetry primer. Adv. Ser. Direct. High Energy Phys. 1998, 18, 1.

6. Djouadi, A. The Anatomy of electro-weak symmetry breaking. II. The Higgs bosons in the minimal supersymmetric model. Phys. Rept. 2008, 1, 459.

7. Kim, J.E.; Nilles, H.P. The mu Problem and the Strong CP Problem. Phys. Lett. B 1984, 138, 150. [CrossRef]

8. Carlos, B.D.; Casas, J.A. One loop analysis of the electroweak breaking in supersymmetric models and the fine tuning problem. Phys. Lett. B 1993, 309, 320. [CrossRef]

9. Chankowski, P.H.; Ellis, J.R.; Pokorski, S. The Fine tuning price of LEP. Phys. Lett. B 1998, 423, 327. [CrossRef] 
10. Barbieri, R.; Strumia, A. About the fine tuning price of LEP. Phys. Lett. B 1998, 433, 63. [CrossRef]

11. Kane, G.L.; King, S.F. Naturalness implications of LEP results. Phys. Lett. B 1999, 451, 113. [CrossRef]

12. Giusti, L.; Romanino, A.; Strumia, A. Natural ranges of supersymmetric signals. Nucl. Phys. B 1999, 550, 3. [CrossRef]

13. Maniatis, M. The next-to-minimal supersymmetric extension of the standard model reviewed. Int. J. Mod. Phys. A 2010, 25, 3505-3602. [CrossRef]

14. Ellwanger, U.; Hugonie, C.; Teixeira, A.M. The next-to-minimal supersymmetric standard model. Phys. Rep. 2010, 496, 1-77. [CrossRef]

15. Ellwanger, U. A Higgs boson near $125 \mathrm{GeV}$ with enhanced di-photon signal in the NMSSM. J. High Energy Phys. 2012, 44 , 1203. [CrossRef]

16. Gunion, J.F.; Jiang, Y.; Kraml, S. The Constrained NMSSM and Higgs near 125 GeV. Phys. Lett. B 2012, 710, 454. [CrossRef]

17. King, S.F.; Muhlleitner, M.; Nevzorov, R. NMSSM Higgs Benchmarks Near 125 GeV. Nucl. Phys. B 2012, 860, 207. [CrossRef]

18. Cao, J.J.; Heng, Z.X.; Yang, J.M.; Zhang, Y.M.; Zhu, J.Y. A SM-like Higgs near 125 GeV in low energy SUSY: A comparative study for MSSM and NMSSM. J. High Energy Phys. 2012, 86, 1203. [CrossRef]

19. Vasquez, D.A.; Belanger, G.; Boehm, C.; da Silva, J.; Richardson, P.; Wymant, C. The 125 GeV Higgs in the NMSSM in light of LHC results and astrophysics constraints. Phys. Rev. D 2012, 86, 35023. [CrossRef]

20. Ellwanger, U.; Hugonie, C. Higgs bosons near $125 \mathrm{GeV}$ in the NMSSM with constraints at the GUT scale. Adv. High Energy Phys. 2012, 2012, 625389. [CrossRef]

21. Benbrik, R.; Bock, M.G.; Heinemeyer, S.; Stal, O.; Weiglein, G.; Zeune, L. Confronting the MSSM and the NMSSM with the Discovery of a Signal in the two Photon Channel at the LHC. Eur. Phys. J. C 2012, 72, 2171. [CrossRef]

22. Gunion, J.F.; Jiang, Y.; Kraml, S. Could two NMSSM Higgs bosons be present near 125 GeV? Phys. Rev. D 2012, 86, 71702. [CrossRef]

23. Bae, K.J.; Choi, K.; Chun, E.J.; Im, S.H.; Park, C.B.; Shin, C.S. Peccei-Quinn NMSSM in the light of 125 GeV Higgs. J. High Energy Phys. 2012, 1211, 118. [CrossRef]

24. Agashe, K.; Cui, Y.; Franceschini, R. Natural Islands for a $125 \mathrm{GeV}$ Higgs in the scale-invariant NMSSM. J. High Energy Phys. 2013, 1302, 31. [CrossRef]

25. Choi, K.; Im, S.H.; Jeong, K.S.; Yamaguchi, M. Higgs mixing and diphoton rate enhancement in NMSSM models. J. High Energy Phys. 2013, 1302, 90. [CrossRef]

26. Kowalska, K.; Munir, S.; Roszkowski, L.; Sessolo, E.M.; Trojanowski, S.; Tsai, Y.-L.S. Constrained next-to-minimal supersymmetric standard model with a $126 \mathrm{GeV}$ Higgs boson: A global analysis. Phys. Rev. D 2013, 87, 115010. [CrossRef]

27. King, S.F.; Muhlleitner, M.; Nevzorov, R.; Walz, K. Natural NMSSM Higgs Bosons. Nucl. Phys. B 2013, 870, 323. [CrossRef]

28. Gherghetta, T.; von Harling, B.; Medina, A.D.; Schmidt, M.A. The Scale-Invariant NMSSM and the 126 GeV Higgs Boson. J. High Energy Phys. 2013, 1302, 32. [CrossRef]

29. Badziak, M.; Olechowski, M.; Pokorski, S. New Regions in the NMSSM with a 125 GeV Higgs. J. High Energy Phys. 2013, $1306,43$. [CrossRef]

30. Gunion, J.F.; Haber, H.E.; Moroi, T. Will at least one of the Higgs bosons of the next-to-minimal supersymmetric extension of the standard model be observable at LEP-2 or the LHC? arXiv 1996, arXiv:hep-ph/9610337.

31. Dobrescu, B.A.; Matchev, K.T. Light axion within the next-to-minimal supersymmetric standard model. J. High Energy Phys. 2000, 0009,31 . [CrossRef]

32. Dobrescu, B.A.; Landsberg, G.L.; Matchev, K.T. Higgs boson decays to CP odd scalars at the Tevatron and beyond. Phys. Rev. D 2001, 63, 075003. [CrossRef]

33. Dermisek, R.; Gunion, J.F. Escaping the large fine tuning and little hierarchy problems in the next to minimal supersymmetric model and $\mathrm{h} \longrightarrow$ aa decays. Phys. Rev. Lett. 2005, 95, 041801. [CrossRef]

34. Moretti, S.; Munir, S. Di-photon Higgs signals at the LHC in the next-to-minimal supersymmetric standard model. Eur. Phys. J. C 2006, 47, 791-803. [CrossRef]

35. Moretti, S.; Munir, S.; Poulose, P. Another step towards a no-lose theorem for NMSSM Higgs discovery at the LHC. Phys. Lett. B 2007, 644, 241-247. [CrossRef]

36. Arhrib, A.; Cheung, K.; Hou, T.J.; Song, K.W. Associated production of a light pseudoscalar Higgs boson with a chargino pair in the NMSSM. J. High Energy Phys. 2007, 703, 73. [CrossRef]

37. Dermisek, R.; Gunion, J.F. A Comparison of Mixed-Higgs Scenarios In the NMSSM and the MSSM. Phys. Rev. D 2008, 77, 015013. [CrossRef]

38. Cheung, K.; Hou, T.J. Light Pseudoscalar Higgs boson in Neutralino Decays in the Next-to-Minimal Supersymmetric Standard Model. Phys. Lett. B 2009, 674, 54. [CrossRef]

39. Lisanti, M.; Wacker, J.G. Discovering the Higgs boson with low mass muon pairs. Phys. Rev. D 2009, 79, 115006. [CrossRef]

40. Belyaev, A.; Pivarski, J.; Safonov, A.; Senkin, S.; Tatarinov, A. LHC discovery potential of the lightest NMSSM Higgs boson in the $h_{1} \rightarrow a_{1} a_{1} \rightarrow 4 \mu$ channel. Phys. Rev. D 2010, 81, 075021. [CrossRef]

41. Mahmoudi, F.; Rathsman, J.; Stal, O.; Zeune, L. Light Higgs bosons in phenomenological NMSSM. Eur. Phys. J. C 2011, 71, 1608. [CrossRef]

42. Almarashi, M.M.; Moretti, S. Low Mass Higgs signals at the LHC in the Next-to-Minimal Supersymmetric Standard Model. Eur. Phys. J. C 2011, 71, 1618. [CrossRef] 
43. Almarashi, M.M.; Moretti, S. Very Light CP-odd Higgs bosons of the NMSSM at the LHC in 4b-quark final states. Phys. Rev. D 2011, 84, 15014. [CrossRef]

44. Almarashi, M.M.; Moretti, S. Muon Signals of Very Light CP-odd Higgs states of the NMSSM at the LHC. Phys. Rev. D 2011, 83, 35023. [CrossRef]

45. Belanger, G.; Ellwanger, U.; Gunion, J.F.; Jiang, Y.; Kraml, S.; Schwarz, J.H. Higgs Bosons at 98 and 125 GeV at LEP and the LHC. J. High Energy Phys. 2013, 1, 069. [CrossRef]

46. Cerde no, D.G.; Ghosh, P.; Park, C.B.; Peiro, M. Collider signatures of a light NMSSM pseudoscalar in neutralino decays in the light of LHC results. J. High Energy Phys. 2014, 1402, 48. [CrossRef]

47. King, S.F.; Muhlleitner, M.; Nevzorov, R.; Walz, K. Discovery Prospects for NMSSM Higgs Bosons at the High-Energy Large Hadron Collider. Phys. Rev. D 2014, 90, 095014. [CrossRef]

48. Bomark, N.E.; Moretti, S.; Munir, S.; Roszkowski, L. A light NMSSM pseudoscalar Higgs boson at the LHC redux. J. High Energy Phys. 2015, 1502, 44. [CrossRef]

49. Ellwanger, U.; Hugonie, C. A $750 \mathrm{GeV}$ Diphoton Signal from a Very Light Pseudoscalar in the NMSSM. J. High Energy Phys. 2016, 1605, 114. [CrossRef]

50. Conte, E.; Fuks, B.; Guo, J.; Li, J.; Williams, A.G. Investigating light NMSSM pseudoscalar states with boosted ditau tagging. J. High Energy Phys. 2016, 1605, 100. [CrossRef]

51. Guchait, M.; Kumar, J. Diphoton Signal of light pseudoscalar in NMSSM at the LHC. Phys. Rev. D 2017, 3, 35036. [CrossRef]

52. Ellwanger, U.; Rodriguez-Vazquez, M. Discovery Prospects of a Light Scalar in the NMSSM. J. High Energy Phys. 2016, 96, 1602. [CrossRef]

53. Domingo, F. Decays of a NMSSM CP-odd Higgs in the low-mass region. J. High Energy Phys. 2017, 52, 1703. [CrossRef]

54. Almarashi, M.M. Prospects of Neutral Higgs boson decays in the NMSSM. Results Phys. 2018, 9, 534. [CrossRef]

55. Almarashi, M.M. Discovery potential of the NMSSM CP-odd Higgs at the LHC. Results Phys. 2018, 10, 799. [CrossRef]

56. Almarashi, M.M. Light CP-odd Higgs boson production of the NMSSM in bottom-gluon fusion at the LHC. Int. J. Mod. Phys. A 2020, 25, 2050151. [CrossRef]

57. Almarashi, M.M. Detection prospects of a low-mass pseudoscalar Higgs in bottom-quark fusion in the NMSSM at the LHC. Results Phys. 2021, 23, 104022. [CrossRef]

58. Almarashi, M.M. LHC signals of the next-to-lightest scalar Higgs state of the NMSSM in the $4 \tau$ decay channel. Adv. High Energy Phys. 2021, 2021, 5569862. [CrossRef]

59. Tung, Y.C.; Hsiung, Y.B.; Wu, M.L.; Chen, K.F.; Ahn, J.K.; Akune, Y.; Baranov, V.; Comfort, J.; Doroshenko, M.; Fujioka, Y.; et al Search for a light pseudoscalar particle in the decay K0(L) $\rightarrow$ pi0 pi0 X. Phys. Rev. Lett. 2009, 102, 051802. [CrossRef] [PubMed]

60. Chatrchyan, S. et al. [CMS Collaboration]. Search for a Light Pseudoscalar Higgs Boson in the Dimuon Decay Channel in $p p$ Collisions at $\sqrt{s}=7$ TeV. Phys. Rev. Lett. 2012, 109, 121801. [CrossRef] [PubMed]

61. Lees, J.P. et al. [BABAR Collaboration]. Search for di-muon decays of a low-mass Higgs boson in radiative decays of the Y(1S). Phys. Rev. D 2013, 3, 31102 .

62. Lees, J.P. et al. [BABAR Collaboration]. Search for a low-mass scalar Higgs boson decaying to a tau pair in single-photon decays of Y(1S). Phys. Rev. D 2013, 7, 71102.

63. Aad, G. et al. [ATLAS Collaboration]. Search for a CP-odd Higgs boson decaying to Zh in pp collisions at $\sqrt{s}=8 \mathrm{TeV}$ with the ATLAS detector. Phys. Lett. B 2015, 744, 163. [CrossRef]

64. Sirunyan, A.M. et al. [CMS Collaboration]. Search for a light pseudoscalar Higgs boson produced in association with bottom quarks in pp collisions at $\sqrt{s}=8$ TeV. J. High Energy Phys. 2017, 1711, 10.

65. Sirunyan, A.M. et al. [CMS Collaboration]. Search for an exotic decay of the Higgs boson to a pair of light pseudoscalars in the final state with two b quarks and two $\tau$ leptons in proton-proton collisions at $\sqrt{s}=13$ TeV. Phys. Lett. B 2018, 785, 462. [CrossRef]

66. Seong, I.S. et al. [Belle Collaboration]. Search for a light CP-odd Higgs boson and low-mass dark matter at the Belle experiment. Phys. Rev. Lett. 2019, 122, 011801. [CrossRef]

67. Sirunyan, A.M. et al. [CMS Collaboration]. Search for an exotic decay of the Higgs boson to a pair of light pseudoscalars in the final state with two muons and two b quarks in pp collisions at 13 TeV. Phys. Lett. B 2019, 795, 398. [CrossRef]

68. Sirunyan, A.M. et al. [CMS Collaboration]. Search for light pseudoscalar boson pairs produced from decays of the $125 \mathrm{GeV}$ Higgs boson in final states with two muons and two nearby tracks in pp collisions at $\sqrt{s}=13$ TeV. Phys. Lett. B 2020, 800, 135087 . [CrossRef]

69. Dittmaier, S.; Kramer, M.; Spira, M.; Walser, M. Charged-Higgs-boson production at the LHC: NLO supersymmetric QCD corrections. Phys. Rev. D 2011, 83, 055005. [CrossRef]

70. Degrande, C.; Ubiali, M.; Wiesemann, M.; Zaro, M. Heavy charged Higgs boson production at the LHC. J. High Energy Phys. 2015, 1510, 145. [CrossRef]

71. Dawson, S.; Kao, C.; Wang, Y.; Williams, P. QCD Corrections to Higgs Pair Production in Bottom Quark Fusion. Phys. Rev. D 2007, 75, 013007. [CrossRef]

72. Dittmaier, S.; Kroamer, M.; Muck, A.; Schluter, T. MSSM Higgs-boson production in bottom-quark fusion: Electroweak radiative corrections. J. High Energy Phys. 2007, 0703, 114. [CrossRef]

73. Dawson, S.; Kao, C.; Wang, Y. SUSY QCD Corrections to Higgs Pair Production from Bottom Quark Fusion. Phys. Rev. D 2008, 77, 113005. [CrossRef] 
74. Zhu, H.X.; Li, C.S.; Zhang, J.J.; Zhang, H.; Li, Z. Threshold Resummation Effects in Neutral Higgs Boson Production by Bottom Quark Fusion at the CERN Large Hadron Collider. Phys. Rev. D 2009, 79, 113005. [CrossRef]

75. Harlander, R.V.; Ozeren, K.J.; Wiesemann, M. Higgs plus jet production in bottom quark annihilation at next-to-leading order. Phys. Lett. B 2010, 693, 269. [CrossRef]

76. Bouhler, S.; Herzog, F.; Lazopoulos, A.; Mouller, R. The fully differential hadronic production of a Higgs boson via bottom quark fusion at NNLO. J. High Energy Phys. 2012, 1207, 115. [CrossRef]

77. Harlander, R.V.; Tripathi, A.; Wiesemann, M. Higgs production in bottom quark annihilation: Transverse momentum distribution at NNLO+NNLL. Phys. Rev. D 2014, 90, 15017. [CrossRef]

78. Forte, S.; Napoletano, D.; Ubiali, M. Higgs production in bottom-quark fusion in a matched scheme. Phys. Lett. B 2015, 751, 331. [CrossRef]

79. Forte, S.; Napoletano, D.; Ubiali, M. Higgs production in bottom-quark fusion: Matching beyond leading order. Phys. Lett. B 2016, 763, 190. [CrossRef]

80. Ajjath, A.H.; Banerjee, P.; Chakraborty, A.; Dhani, P.K.; Mukherjee, P.; Rana, N.; Ravindran, V. Higgs pair production from bottom quark annihilation to NNLO in QCD. J. High Energy Phys. 2019, 1905, 30. [CrossRef]

81. Ajjath, A.H.; Banerjee, P.; Chakraborty, A.; Dhani, P.K.; Mukherjee, P.; Rana, N.; Ravindran, V. NNLO QCD $\oplus$ QED corrections to Higgs production in bottom quark annihilation. Phys. Rev. D 2019, 11, 114016.

82. Forte, S.; Giani, T.; Napoletano, D. Fitting the b-quark PDF as a massive-b scheme: Higgs production in bottom fusion. Eur. Phys. J. C 2019, 7, 609. [CrossRef]

83. Duhr, C.; Dulat, F.; Mistlberger, B. Higgs production in bottom-quark fusion to third order in the strong coupling. Phys. Rev. Lett. 2020, 5, 51804. [CrossRef]

84. Duhr, C.; Dulat, F.; Hirschi, V.; Mistlberger, B. Higgs production in bottom quark fusion: Matching the 4- and 5-flavour schemes to third order in the strong coupling. J. High Energy Phys. 2020, 2008, 17. [CrossRef]

85. Peccei, R.D.; Quinn, H.R. CP Conservation in the Presence of Instantons. Phys. Rev. Lett. 1977, 38, 1440. [CrossRef]

86. Peccei, R.D.; Quinn, H.R. Constraints Imposed by CP Conservation in the Presence of Instantons. Phys. Rev. D 1977, 16, 1791. [CrossRef]

87. Miller, D.J.; Nevzorov, R.; Zerwas, P.M. The Higgs sector of the next-to-minimal supersymmetric standard model. Nucl. Phys. B 2004, 681, 3-30. [CrossRef]

88. Baglio, J.; Groober, R.; Mouhlleitner, M.; Nhung, D.T.; Rzehak, H.; Spira, M.; Streicher, J.; Walz, K. NMSSMCALC: A Program Package for the Calculation of Loop-Corrected Higgs Boson Masses and Decay Widths in the (Complex) NMSSM. Comput. Phys. Commun. 2014, 12, 3372. [CrossRef]

89. Belanger, G.; Bizouard, V.; Chalons, G. Boosting Higgs boson decays into gamma and a Z in the NMSSM. Phys. Rev. D 2014, 9 , 095023. [CrossRef]

90. Liebler, S. Neutral Higgs production at proton colliders in the CP-conserving NMSSM. Eur. Phys. J. C 2015, 5, 210. [CrossRef]

91. Liebler, S.; Mantler, H.; Wiesemann, M. Distributions for neutral Higgs production in the NMSSM. arXiv 2012, arXiv:1608.02949.

92. Goodsell, M.D.; Liebler, S.; Staub, F. Generic calculation of two-body partial decay widths at the full one-loop level. Eur. Phys. J. C 2017, 11, 758. [CrossRef]

93. Allanach, B.C.; Cridge, T. The Calculation of Sparticle and Higgs Decays in the Minimal and Next-to-Minimal Supersymmetric Standard Models: SOFTSUSY4.0. Comput. Phys. Commun. 2017, 220, 417. [CrossRef]

94. Elanger, G.B.; Bizouard, V.; Boudjema, F.; Chalons, G. One-loop renormalization of the NMSSM in SloopS. II. The Higgs sector. Phys. Rev. D 2017, 96, 15040. [CrossRef]

95. Domingo, F.; Heinemeyer, S.; Paoehr, S.; Weiglein, G. Decays of the neutral Higgs bosons into SM fermions and gauge bosons in the $\mathcal{C P}$-violating NMSSM. Eur. Phys. J. C 2018, 78, 942. [CrossRef]

96. Domingo, F.; Paßehr, S. Electroweak corrections to the fermionic decays of heavy Higgs states. Eur. Phys. J. C 2019, 11, 905. [CrossRef]

97. Baglio, J.; Dao, T.N.; Mouhlleitner, M. One-Loop Corrections to the Two-Body Decays of the Neutral Higgs Bosons in the Complex NMSSM. Eur. Phys. J. C 2020, 10, 960. [CrossRef]

98. Baglio, J.; Campanario, F.; Glaus, S.; Mouhlleitner, M.; Ronca, J.; Spira, M.; Streicher, J. Higgs-Pair Production via Gluon Fusion at Hadron Colliders: NLO QCD Corrections. J. High Energy Phys. 2020, 80, 181. [CrossRef]

99. Domingo, F.; Paehr, S. Towards Higgs masses and decay widths satisfying the symmetries in the (N)MSSM. Eur. Phys. J. C 2020, 12, 1124. [CrossRef]

100. Baglio, J.; Campanario, F.; Glaus, S.; Mouhlleitner, M.; Ronca, J.; Spira, M. gg $\rightarrow H H$ : Combined uncertainties. Phys. Rev. D 2021, 5, 56002. [CrossRef]

101. Dermisek, R.; Gunion, J.F. Next-to-minimal supersymmetric model solution to the fine-tuning problem, precision electroweak constraints, and the largest CERN LEP Higgs event excess. Phys. Rev. D. 2007, 76, 95006. [CrossRef]

102. Ellwanger, U.; Gunion, J.F.; Hugonie, C. NMHDECAY: A Fortran Code for the Higgs Masses, Couplings and Decay widths in the NMSSM. 2005. Available online: https:/ /iopscience.iop.org/article/10.1088/1126-6708/2005/02/066/pdf (accessed on 18 August 2020).

103. Ellwanger, U.; Hugonie, C. NMHDECAY 2.1: An updated program for sparticle masses, Higgs masses, couplings and decay widths in the NMSSM. Comput. Phys. Commun. 2006, 175, 290-303. [CrossRef] 
104. NMSSMTOOLS-Home. Available online: https://www.lupm.univ-montp2.fr/users/nmssm/ (accessed on 18 August 2020).

105. Domingo, F. A New Tool for the study of the CP-violating NMSSM. J. High Energy Phys. 2015, 6, 052. [CrossRef]

106. Belyaev, A.; Christensen, N.D.; Pukhov, A. CalcHEP 3.4 for collider physics within and beyond the Standard Model. Comput. Phys. Commun. 2013, 184, 1729. [CrossRef]

107. Andreas, S.; Lebedev, O.; Sanchez, S.R.; Ringwald, A. Constraints on a very light CP-odd Higgs of the NMSSM and other axion-like particles. J. High Energy Phys. 2010, 1008, 003. [CrossRef]

108. Almarashi, M.M. Study of a Light NMSSM CP-Odd Higgs Produced via Bottom-Quark Annihilation in the Di-Photon Channel at the LHC. 2021. Available online: http:/ / www.andromedapublisher.com/media/img/confprocimg/3/Pdf/MoslehAlmarashi_ vqY8tCJ.pdf (accessed on 10 October 2021).

109. OPAL Collaboration. Decay mode independent searches for new scalar bosons with the OPAL detector at LEP. Eur. Phys. J. C 2003, 27, 311. [CrossRef]

110. LEP Working Group for Higgs Boson Searches. ALEPH, DELPHI, L3 and OPAL. Search for the standard model Higgs boson at LEP. Phys. Lett. B 2003, 565, 61. [CrossRef]

111. ALEPH, DELPHI, L3, OPAL and LEP Working Group for Higgs Boson Searches. Search for neutral MSSM Higgs bosons at LEP. Eur. Phys. J. C 2006, 47, 547. [CrossRef]

112. Tevatron New Physics Higgs Working Group. Updated Combination of CDF and D0 Searches for Standard Model Higgs Boson Production with up to $10.0 \mathrm{fb}^{-1}$ of Data. arXiv 2012, arXiv:1207.0449.

113. Search for Resonances in the 65 to $110 \mathrm{GeV}$ Diphoton Invariant Mass Range Using $80 \mathrm{fb}^{-1}$ of pp Collisions Collected at $\sqrt{s}=13 \mathrm{TeV}$ with the ATLAS Detector. Available online: https://cds.cern.ch/record/2628760/files/ATLAS-CONF-2018-025.pdf (accessed on 10 October 2021).

114. Canelli, M.F. et al. [CMS Collaboration]. Search for additional neutral MSSM Higgs bosons in the $\tau \tau$ final state in proton-proton collisions at $\sqrt{s}=13 \mathrm{TeV}$. J. High Energy Phys. 2018, 9, 7.

115. Sirunyan, A.M. et al. [CMS Collaboration]. Search for a standard model-like Higgs boson in the mass range between 70 and 110 $\mathrm{GeV}$ in the diphoton final state in proton-proton collisions at $\sqrt{s}=8$ and 13 TeV. Phys. Lett. B 2019, 793, 320. [CrossRef] 